\title{
Recurring Severe Injection-related Infections in People Who Inject Drugs and the Need for Safe Injection Sites in Spain
}

Jorge Valencia ( $\sim$ jorge_vlr@yahoo.es )

Servicio movil de reducción del daño SMASD; Subdirección General de Adicciones

https://orcid.org/0000-0002-0148-2422

Jesús Troya

Hospital Infanta Leonor

Jeffrey V Lazarus

Barcelona Institute for Global Health: Instituto de Salud Global Barcelona

Guillermo Cuevas

Hospital Infanta Leonor

Alejandro Alvaro-Meca

Universidad Rey Juan Carlos

Juan Torres-Macho

Hospital Infanta Leonor

Carlos Gardeta

Harm Reduction Unit SMASD

David Lozano

Harm Reduction Unit SMASD

Santiago Moreno

Ramon y Cajal University Hospital: Hospital Universitario Ramon y Cajal

Pablo Ryan

Hospital Infanta Leonor

\section{Research}

Keywords: Skin and soft tissue infections, harm reduction, opioid agonist therapy, safe injection sites, severe injection-related infections

Posted Date: April 7th, 2021

DOl: https://doi.org/10.21203/rs.3.rs-389593/v1 
License: (c) (i) This work is licensed under a Creative Commons Attribution 4.0 International License. Read Full License 


\section{Abstract}

Background and aims: An estimated 68,297 people with opioid use disorder engage in opioid agonist therapy (OAT) in Spain. We aimed to calculate the incidence of severe injection-related infections in people who inject drugs (PWID) engaged in OAT in a harm reduction setting without a safe injection site (SCS).

Methods: A retrospective cohort study was performed in PWID engaged in OAT and in a mobile harm reduction unit to identify who was admitted to a referral hospital for any severe injection-related infections between 1 Jan 2016 and 31 Dec 2019. A Cox proportional hazard regression analysis was used to assess factors associated with any severe injection-related infection.

Results: 237 PWID engaged in OAT were included. After a median follow-up of 5.5 months (IQR 1.322.7), a total of 104 episodes of severe injection-related infections occurred in 56 individuals, and admission for a second event occurred in $35.7 \%$ of this same group. The incidence density of any type of severe injection-related infection was 26.8 (20.2-34.8) episodes per $100 \mathrm{PY}$, and the incidence density of complicated skin and soft tissue infections (SSTIs) that required hospital admission was 20.4 (15.027.3) episodes per 100 PY. Fifty-six (53.8\%) of all the episodes were patient-directed discharge (PDD), and people who had two or more hospital admissions had a higher PDD frequency.

Conclusion: Severe injection-related infections remain highly prevalent among PWID cared for in a harm reduction setting without a SCS. PDD were more frequent in higher-risk individuals who presented two or more hospital readmissions.

\section{Background}

People who inject drugs (PWID) present at emergency departments more often than the general population $(1,2)$. Sharing of equipment causes a substantial disease burden in PWID, and bacterial and viral infections are largely responsible for the significant mortality and morbidity of the PWID presenting to secondary care (3). The delays in seeking health care, barriers to accessing care, and interruptions of skin and soft tissue infection (SSTI) therapy and the subsequent systemic progression might increase costs and mortality in people with an SSTI.

PWID with IDU-associated infections also have a high frequency of patient-directed discharge (PDD) without completing treatment (4). Additionally, a recent study revealed that those who did not receive oral antibiotics upon PDD had a 90-day readmission rate, which is more than double the rate of those with PDD who did have an antibiotic prescribed (5). Another recent study reported that opioid/stimulant use was associated with a higher risk of PDD and in-hospital mortality compared to opioid-only use (6).

Current data suggest that the number of hospital admissions for injecting-related bacterial infections is increasing in some regions $(7,8)$. In this sense, safe injection sites (SCSs) have been proven to increase access to safer injection conditions, reduce drug use in public spaces, lower overdose rates, link users to 
healthcare, and reduce fear and stigmatization (9-12). Also, SCSs have been shown to be a costeffective public health strategy that reduces treatment costs associated with new HIV and hepatitis $\mathrm{C}$ virus (HCV) infections, wound care, and overdose-related services $(13,14)$.

Harm reduction services in Spain are provided in the different autonomous communities by a large network of public facilities, including social emergency centers, mobile units, pharmacies, and prisons, and are available throughout the country. Thirteen facilities for supervised drug consumption are available in the autonomous communities of Catalonia and the Basque Country (15). However, Madrid closed a van that offered a drug consumption mobile space integrated into a mobile harm reduction unit in 2009 and currently does not provide safe injection facilities.

There are few real-world studies with standardized definitions and documented clinical events calculating the incidence and predictors for hospital admission for severe bacterial infections among PWID without access to safe injection facilities (16). Thus, our objective was to estimate the incidence of any severe injection-related infections among PWID on opioid agonist therapy (OAT) attending a harm reduction setting. We hypothesized that hospitalizations for any severe injection-related infections in PWID on OAT would be higher than prior reports published in the literature because this mobile harm reduction unit lacks safe spaces for drug consumption.

\section{Methods}

\section{Study population}

This retrospective, observational study is based on data from a follow-up cohort of people engaged in OAT who inject cocaine and heroin actively in the last six months and were attending a mobile harm reduction unit located in the outskirts of Madrid between 1 January 2016 to 30 December 2019. This mobile unit is based on a low-threshold approach consisting of no entry requirements, no appointments, a non-judgmental nature, flexibility, and on-site healthcare on demand.

\section{Setting}

This study was conducted in a mobile harm reduction unit, where a comprehensive, multidisciplinary team actively cared for and followed PWID with limited access to standard healthcare. Several services were offered, including needle-exchange programs (NEP) consisting of safe disposal for used needles, distributing naloxone, OAT, frequent testing for infectious diseases, dispensing treatment for chronic diseases, harm reduction education, counseling, and social support on-demand and as low-threshold. This mobile harm reduction unit has five vans used by health workers and offers different services. Despite these provisions, due to the absence of mobile safe injection rooms, people inject alone, in pairs, or groups in the street, cars, parks, tents, accommodations, or in their houses.

In Madrid, people with opioid use disorder engaged in harm reduction services are dispensed liquid formulations of methadone hydrochloride and no other opiate substitutes are allowed in this setting in 
order to avoid illegal sale or misuse of opiate pills. However, when individuals are in inpatient hospital care, all individuals continue methadone treatment and doses are increased to overcome opiate cravings.

\section{Methodology}

Registered clinical data at the mobile harm reduction unit were reviewed to identify cases of severe bacterial injection-related infections that required hospital admission in people on OAT (discharge summaries through standardized coding system (ICD-10)). Information related to demographic data, retention in OAT, and type of SSTI was collected from medical records, as well as local electronic data systems at the mobile harm reduction unit. The clinical information and outcomes of each event were collected from the electronic medical records at the hospital.

Healthcare personnel at the mobile harm reduction unit interviewed each participant with a short questionnaire when they started OAT and recorded their drug use and clinical data. Information regarding the type of drug use and route of use was collected and based on self-reports.

\section{Case definitions}

Severe injection-related infections were defined as the presence of any of the following types of IDUrelated infections that required hospital admission and occurred during the follow-up: complicated SSTI, bacterial isolates from the blood (bacteremia), infective endocarditis (IE), or bacteremia with spread to other non-cardiac locations.

Complicated SSTIs were defined as cases of skin abscesses or ulcers, extended cellulitis, myositis, or fasciitis at the injecting site. An episode of IE diagnosed at the hospital was defined as definite or possible according to the modified Duke criteria (17). Bacteremia was defined as an isolation of any bacteria from the bloodstream obtained through blood culture systems in conjunction with fever and other symptoms of infection without conclusive evidence of disseminated infection to other organs. Bacteremia with spread to other non-cardiac locations was defined by an isolate of any bacteria considered clinical and microbiologic, related to IDU, with hematogenous spread to other non-cardiac organs, such as discitis, arthritis, septic emboli, osteomyelitis, or spondylitis.

Hospital readmissions that occurred within 90 days from the discharge date were considered as relapses and not a new episode of an SSTI. However, if the readmission occurred within the first 90 days from discharge and the microorganism in the blood culture was different from the previous episode, it was classified as a new event. All the readmissions for the same person after day 90 from hospital discharge were considered as new episodes of SSTIs and recorded as separate events.

Discharges from the hospital were classified as medical advice or PDD, and no data were available to describe how many days of antibiotics were missed by PDD.

For this analysis, the identification of pathogens was verified by bloodstream culture results identified from at least one sterile site culture or obtained through a surgical procedure. The events of severe 
injection-related infections with the isolation of Staphylococcus aureus were classified in methicillinsensitive (MSSA) or methicillin-resistant (MRSA).

To calculate the length of stay at the hospital, we considered the admission date for any severe injectionrelated infections and the discharge date for planned discharges or PDD, or the death date in those cases with a fatal outcome. If readmissions due to a relapse occurred, only the first hospital admission was considered.

\section{Statistical analysis}

Categorical variables were compared using the Pearson chi-squared test or Fisher exact test. Continuous variables were compared using the Wilcoxon rank-sum test for independent variables. The Kaplan-Meier method was used to estimate the overall incidence density and incidence density of severe injectionrelated infections according to the type of infections. The time at risk for any severe injection-related infection was calculated from the date of initiation in OAT at the mobile harm reduction unit to the date of hospital admission for any severe injection-related infections or the date last in OAT. Participants remaining persistently free of severe injection-related infections were censored at the time of their last day in OAT prior to 30th December 2019.

Cox proportional hazard regression analysis was used to assess factors associated with time to event of severe injection-related infection. In the unadjusted analyses, potential predictors were determined according to previous reports and included: age (per year), sex, nationality, mental health illness, HIV and $\mathrm{HCV}$ infection. All variables with $\mathrm{p}<0.05$ in the univariate analysis were included in the multivariate regression models using an Akaike Information Criterion (AIC) method approach. Statistical significance was set at $p<0.05 ; p$-values were two-sided.

Study data were collected and managed using REDCap (Research Electronic Data Capture) tools hosted at "Asociación ideas for Health" (18). REDCap is a web-based software platform designed to support data capture for research studies. Analyses were performed using R software (R Foundation, Vienna, Austria).

\section{Results}

A total of 479 individuals who used drugs actively engaged on OAT were followed between 1 January 2016 and 30 December 2019 at the mobile harm reduction unit. Of those, 237 reported recent injection drug use and they were included in the study for calculating the incidence density of severe injectionrelated infections. All 237 individuals self-reported use of injected heroin and cocaine mixed in different non-quantified proportions (stimulant-only use was not reported). The demographic characteristics are outlined in Table 1.

Overall, we found that $160(67.5 \%)$ individuals included in the study period presented in follow-up with at least one [medium range $4.5(1-8)$ ] non-complicated SSTI that did not require hospitalization and they were treated with oral antibiotics at the mobile harm reduction unit. Severe injection-related infections 
were registered in 56 individuals and accounted for 104 episodes. Therefore, the global incidence density for any severe injection-related infection was 26.8 (20.2-34.8) episodes per 100 person years (PY) (Fig. 1). According to the type of severe injection-related infection, there were eight episodes of bacteremia with an incidence density of 3.0 (1.3-6.0) episodes per $100 \mathrm{PY}$; six episodes of IE with an incidence density of $2.3(0.8-5.0)$ episodes per 100 PY; 19 episodes of bacteremia plus any infection with a non-cardiac location with an incidence density of 7.7 (4.6-12.0) episodes per 100 PY; and 64 episodes of complicated SSTIs with an incidence density of 20.4 (15.0-27.3) episodes per 100 PY(Fig. 2).

The recurrence or a new event of severe injection-related infection occurred in 20 (35.7\%) of the 56 individuals who had had a previous severe infection, eight (44.6\%) of them with at least three episodes and four individuals in all of the cohort with four or more episodes of severe injection-related infections in the follow-up. Episodes of relapses with readmissions to hospital occurred in 11 (19.6\%) individuals during follow-up.

All episodes of IE met the Duke criteria and were caused by Gram-positive bacteria. The most frequent sites of SSTIs were: limbs, 29 (45.3\%); arms, 18 (28.1\%); groin, ten (15.6\%); hands, four (6.3\%); and neck, three $(4.7 \%)$. Five $(7.8 \%)$ events of complicated SSTIs required surgical management.

The median hospital length of stay was six days (IQR 2.0-11.0); however, 56 (53.8\%) of all episodes of severe injection-related infections were PDD and did not complete antibiotic therapy. People who had two or more hospital admissions for IDU-related bacterial infections had more frequency of PDD $(p<0.001)$ in comparison to people who had a unique hospital admission.

The microorganisms responsible for severe injection-related infections were predominantly Staphylococcus aureus [32 (55.2\%) cases], Streptococcal species that included $\beta$-hemolytic streptococci [24 (41.4\%) cases], and other Staphylococcus species [two (3.4\%) cases]. MSSA and MRSA were found in $30(93.7 \%)$ and two (6.3\%) episodes of complicated SSTIs, respectively.

Socio-demographic variables, history of mental illness, and HIV and HCV status were not associated with time to a new event of any severe injection-related infection in the adjusted and unadjusted analysis.

During a median follow-up of 5.5 months (IQR 1.3-22.7), there was only one in-hospital death caused by cardiac failure, in the context of an IE.; however, five additional deaths occurred during the follow-up.

\section{Discussion}

Our findings demonstrate that complicated SSTIs and other severe injection-related infections remain highly prevalent and recurring among people injecting stimulants/opiates who are actively cared for in a harm reduction setting. Our results show that despite engagement in OAT and NEP, these incidence rates are even higher than those reported in previous studies of opiate-related, severe bacterial infections in this population (19-22) when harm reduction services did not offer SCSs. 
Notably, at follow-up, around a quarter of the cohort required a hospital admission for any severe injection-related infection that occurred during their remaining time on OAT at the mobile harm reduction unit. Recurrences or new events of severe injection-related infection occurred in more than a third of individuals who had a first hospital admission related to any severe injection-related infection.

Furthermore, a small subgroup of them had three or more recurring episodes of hospital admissions. The IDU-related infections may be explained by daily injecting and engaging in unsafe injecting practices (23, 24), which can contribute to new or repetitive episodes of severe injection-related infections in PWID (25, 26). We also found that people who had two or more hospital admissions for IDU-related bacterial infections had a higher frequency of PDD, and we hypothesized that there is a subgroup of individuals who have higher-risk behaviors, greater severe dependence to stimulant drugs than opiate drugs, and intense craving symptoms, which could explain the recurring events of severe injection-related infections with hospital admissions. Unfortunately, we did not collect data on IDU practices to explore these findings.

In addition, many studies demonstrate that SSTIs are one of the most common reasons for emergency department admissions and hospitalizations in PWID $(23,27)$, with Staphylococcus aureus as a leading cause of SSTIs in PWID (28). However, there are few current and non-cross-sectional studies that investigate the incidence for such bacterial infections among PWID (16). Indeed, our data show that complicated SSTIs were the most frequent cause of admission for severe injection-related infections and occurred in a fifth of participants during their follow-up. Strikingly, these rates are higher compared to previous studies carried out among PWID who attended drug treatment services, outreach programs, and prison and probation services $(19,21,22)$. Rates are only comparable with a previously published report of the incidence of visits to the emergency department for SSTIs among PWID (29). However, our cohort only included episodes of hospitalization and visits to the emergency department was not a variable collected for this study.

This study also highlights that more than two-thirds of the individuals that presented for follow-up had at least one non-complicated SSTI diagnosed and treated at the mobile harm reduction unit that did not required hospitalization. Additionally, their recurrence was very frequent, with individuals having up to eight episodes of non-complicated SSTIs during their follow-up. These rates are about twice as high as those reported in past studies examining SSTIs in $\operatorname{NEP}(19,30)$. This variation is likely due to differing aspects including demographic characteristics and higher-risk injecting behaviors, but we speculate that the absence of SCSs could partly explain these high rates. In the last years, a few studies show similar rates of non-complicated SSTIs $(25,31)$, but these studies were based on self-reported SSTIs or conducted by qualitative interviews and not clinically documented, resulting in bias.

High frequencies of PDD and the use of illicit substances while hospitalized have been reported in previous studies $(33,34,4)$. In our study, we found that at least half of hospital discharges due to severe injection-related infections were PDD. As a probable direct consequence, almost $20 \%$ of individuals who had a hospital admission presented with episodes of relapse with readmission to the hospital. These rates of PDD are higher than in other studies examining people who inject opiates/stimulants or opiates- 
only (35). This can be partially explained by the lack of highly effective medication for stimulant use disorder, which does exist for opioid use disorder, the limited expertise in management of stimulant use disorder by doctors in hospital, and by not using a low-threshold approach during hospitalization.

\section{Strengths and limitations}

The strengths of our study include that we had a homogenous sample of PWID actively using, that infections were clinically documented and registered during the follow-up in OAT, and that we had complete data on hospitalizations and deaths. In comparison, clinical data resources for calculating the incidence of IDU-related bacterial infections among PWID in traditional, previous cohort studies include large-scale databases or regional or national registers using international classification codes of diseases or based on self-reports. The former has inherent limitations: there is no code specifically for IDU, no high validity, no reliable samples that include former IDU and different injected substances, and this population is not exclusively cared for in harm reduction services.

This study is also subject to several limitations. First, this study had a short-term follow-up and it is possible that unsafe injection behaviors decreased over time; therefore, it could be overestimating the incidence of severe injecting-related infections. Second, we defined relapses as happening within 90 days of a previous episode of severe injection-related infection (36), which could cause us to underestimate the number of new events of severe injection-related infections. Third, this is a retrospective study carried out using data from a unique hospital, and it is possible that some of the PWID attended other hospitals in the region or in other regions of Spain. However, since all cases of severe injection-related infections found at our mobile harm reduction unit were derived to this referral hospital due to the geographic location of the health system in Madrid, the likelihood of cases of missed infections is small. Fourth, IDU data were initially collected by self-reporting at the moment of initiating OAT, meaning that it could be affected by self-reporting bias. Although, this information was confirmed through a medical record review and checking of NEP.

\section{Conclusion}

The incidence of severe injection-related infections that occurred among PWID on OAT in a harm reduction setting without a safe injection site was very high and the recurrence of non-complicated SSTIs was highly prevalent. Hospital readmissions for a new event of severe injection-related infection were frequent in a subgroup of higher-risk individuals who also presented more frequency of patient-directed discharge.

\section{Abbreviations}

PWID: people who inject drugs

OAT: opioid agonist therapy 
PDD: patient-directed discharge

SCS: safe injection site

SSTI: skin and soft tissue infection

HCV: hepatitis C virus

HIV: human immunodeficiency virus

NEP: needle-exchange programs

IDU: injection drug use

IE: infective endocarditis

MSSA: methicillin-sensitive Staphylococcus aureus

MRSA: methicillin-resistant Staphylococcus aureus

PY: person years

\section{Declarations}

\section{Ethics approval and consent to participate}

The study was conducted following the Declaration of Helsinki. Data from study participants were anonymized with an alphanumeric code that was unique for each individual. Thus, participants could not be identified and linked to the registered information. When being admitted to the LTMHRU and before start OAT, all individuals signed various documents, including informed consents for blood tests and forms for standard follow-up and analysis of data at the LTMHRU.

The Institutional Investigation and Ethics Review Board of Infanta Leonor University Hospital (CEI-ILUH) approved the study (code ILUH R 038-20) on 23 April 2020.

\section{Consent for publication}

Consent for publication has been granted.

\section{Availability of data and materials}

Datasets used and analyzed during the current study may be available from the "Subdireccion General de Adicciones" (Madrid, Spain) Institutional Data Access for researchers who meet the criteria for confidential data.

\section{Competing interests}


The authors declare they have no competing interests.

\section{Funding}

This work has not been funded.

\section{Author contributions}

Study concept and design: JV, PR and SM.

Patient selection and clinical data acquisition: PR, JV, CG, JT, GC and DL.

Statistical analysis and interpretation of data: AAM.

Drafting the manuscript: JV, PR, JL, and SM.

Critical revision of the manuscript for relevant intellectual content: JV, PR, JT, GC, JL and SM.

Supervision and visualization: JV, PR and SM.

All authors read and approved the final manuscript.

\section{Acknowledgments}

We acknowledge patients' involvement in this study and the NGO "Madrid Positivo." The authors thank Danielle for writing assistance in the preparation of the manuscript.

\section{Originality}

The authors ensure that they have written entirely original works.

\section{Availability of data and materials}

Datasets used and analyzed during the current study may be available from the "Subdireccion General de Adicciones" (Madrid, Spain) Institutional Data Access for researchers who meet the criteria for confidential data.

\section{Acknowledgments}

We acknowledge patients' involvement in this study and the NGO "Madrid Positivo."

JVL acknowledges support to ISGlobal from the Spanish Ministry of Science, Innovation and Universities through the "Centro de Excelencia Severo Ochoa 2019-2023" Programme (CEX2018-000806-S), and from the Government of Catalonia through the CERCA Programme."

\section{References}


1. Milloy MJ, Wood E, Reading C, Kane D, Montaner J, Kerr T. Elevated overdose mortality rates among First Nations individuals in a Canadian setting: a population-based analysis. Addiction. 2010;105(11):1962-70.

2. Degenhardt L, Bucello C, Mathers B, Briegleb C, Ali H, Hickman M, et al. Mortality among regular or dependent users of heroin and other opioids: a systematic review and meta-analysis of cohort studies. Addiction (Abingdon, England). 2011;106(1):32-51.

3. Phillips KT, Anderson BJ, Herman DS, Liebschutz JM, Stein MD. Risk factors associated with skin and soft tissue infections among hospitalized people who inject drugs. Journal of addiction medicine. 2017;11(6):461.

4. Summers PJ, Hellman JL, MacLean MR, Rees VW, Wilkes MS. Negative experiences of pain and withdrawal create barriers to abscess care for people who inject heroin. A mixed methods analysis. Drug Alcohol Depend. 2018; 190:200-8.

5. Marks LR, Liang SY, Muthulingam D, Schwarz ES, Liss DB, Munigala S, et al. Evaluation of partial oral antibiotic treatment for persons who inject drugs and are hospitalized with invasive infections. Clinical Infectious Diseases. 2020.

6. Serota DP, Bartholomew TS, Tookes HE. Evaluating differences in opioid and stimulant useassociated infectious disease hospitalizations in Florida, 2016-2017. Clinical Infectious Diseases. 2020.

7. Ciccarone D, Unick GJ, Cohen JK, Mars SG, Rosenblum D. Nationwide increase in hospitalizations for heroin-related soft tissue infections: Associations with structural market conditions. Drug and Alcohol Dependence. 2016; 163:126-33.

8. Lewer D, Harris M, Hope V. Opiate injection-associated skin, soft tissue, and vascular infections, England, UK, 1997-2016. Emerging infectious diseases. 2017;23(8):1400.

9. Potier C, Laprevote V, Dubois-Arber F, Cottencin O, Rolland B. Supervised injection services: what has been demonstrated? A systematic literature reviews. Drug Alcohol Depend. 2014; 145:48-68.

10. Ng J, Sutherland C, Kolber MR. Does evidence support supervised injection sites? Canadian family physician Medecin de famille canadien. 2017;63(11):866.

11. Bardwell G, Strike C, Altenberg J, Barnaby L, Kerr T. Implementation contexts and the impact of policing on access to supervised consumption services in Toronto, Canada: a qualitative comparative analysis. Harm Reduct J. 2019;16(1):30.

12. Belackova V, Salmon AM, Schatz E, Jauncey M. Drug consumption rooms (DCRs) as a setting to address hepatitis $\mathrm{C}$ - findings from an international online survey. Hepatology, medicine and policy. 2018; 3:9.

13. Irwin A, Jozaghi E, Weir BW, Allen ST, Lindsay A, Sherman SG. Mitigating the heroin crisis in Baltimore, MD, USA: a cost-benefit analysis of a hypothetical supervised injection facility. Harm Reduction Journal. 2017;14(1):29.

14. Jozaghi E, Reid AA, Andresen MA. A cost-benefit/cost-effectiveness analysis of proposed supervised injection facilities in Montreal, Canada. Substance Abuse Treatment, Prevention, and Policy. 
2013;8(1):25.

15. Wiessing $L$, Ferri $M$, Běláčková $V$, Carrieri $P$, Friedman SR, Folch $C$, et al. Monitoring quality and coverage of harm reduction services for people who use drugs: a consensus study. Harm reduction journal. 2017;14(1):19-.

16. Larney S, Peacock A, Mathers BM, Hickman M, Degenhardt L. A systematic review of injecting-related injury and disease among people who inject drugs. Drug Alcohol Depend. 2017; 171:39-49.

17. Li JS, Sexton DJ, Mick N, Nettles R, Fowler VG, Jr., Ryan T, et al. Proposed modifications to the Duke criteria for the diagnosis of infective endocarditis. Clinical infectious diseases: an official publication of the Infectious Diseases Society of America. 2000;30(4):633-8.

18. Harris PA, Taylor R, Thielke R, Payne J, Gonzalez N, Conde JG. Research electronic data capture (REDCap) - a metadata-driven methodology and workflow process for providing translational research informatics support. Journal of biomedical informatics. 2009;42(2):377-81.

19. Hope V, Kimber J, Vickerman P, Hickman M, Ncube F. Frequency, factors and costs associated with injection site infections: Findings from a national multi-site survey of injecting drug users in England. BMC Infectious Diseases. 2008;8(1):120.

20. Lloyd-Smith E, Wood E, Zhang R, Tyndall MW, Montaner JS, Kerr T. Risk factors for developing a cutaneous injection-related infection among injection drug users: a cohort study. BMC public health. 2008;8(1):1-6.

21. Lewer D, Hope VD, Harris M, Kelleher M, Jewell A, Pritchard M, et al. Incidence and treatment costs of severe bacterial infections among people who inject heroin: A cohort study in South London, England. Drug Alcohol Depend. 2020; 212:108057.

22. Dahlman D, Berge J, Björkman P, Nilsson AC, Håkansson A. Both localized and systemic bacterial infections are predicted by injection drug use: a prospective follow-up study in Swedish criminal justice clients. PloS one. 2018;13(5): e0196944.

23. Phillips KT, Anderson BJ, Herman DS, Liebschutz JM, Stein MD. Risk Factors Associated with Skin and Soft Tissue Infections Among Hospitalized People Who Inject Drugs. Journal of addiction medicine. 2017;11(6):461-7.

24. Summers PJ, Struve IA, Wilkes MS, Rees VW. Injection-site vein loss and soft tissue abscesses associated with black tar heroin injection: A cross-sectional study of two distinct populations in USA. The International journal on drug policy. 2017; 39:21-7.

25. Phillips KT, Anderson BJ, Herman DS, Liebschutz JM, Stein MD. Risk Factors Associated with Skin and Soft Tissue Infections Among Hospitalized People Who Inject Drugs. J Addict Med. 2017;11(6):461-7.

26. Eaton EF, Westfall AO, McClesky B, Paddock CS, Lane PS, Cropsey KL, et al., editors. In-Hospital Illicit Drug Use and Patient-Directed Discharge: Barriers to Care for Patients with Injection-Related Infections. Open forum infectious diseases; 2020: Oxford University Press US.

27. Marks M, Pollock E, Armstrong M, Morris-Jones S, Kidd M, Gothard P, et al. Needles and the damage done: reasons for admission and financial costs associated with injecting drug use in a Central 
London Teaching Hospital. Journal of Infection. 2013;66(1):95-102.

28. Lloyd-Smith E, Hull MW, Tyndall MW, Zhang R, Wood E, Montaner JSG, et al. Community-associated methicillin-resistant Staphylococcus aureus is prevalent in wounds of community-based injection drug users. Epidemiology and infection. 2010;138(5):713-20.

29. Lloyd-Smith E, Tyndall M, Zhang R, Grafstein E, Sheps S, Wood E, et al. Determinants of cutaneous injection-related infections among injection drug users at an emergency department. The open infectious diseases journal. 2012;6.

30. Dahlman D, Håkansson A, Björkman P, Blomé MA, Kral AH. Correlates of skin and soft tissue infections in injection drug users in a syringe-exchange program in Malmö, Sweden. Substance Use \& Misuse. 2015;50(12):1529-35.

31. Doran J, Harris M, Hope VD, Wright T, Edmundson C, Sinka K, et al. Factors associated with skin and soft tissue infections among people who inject drugs in the United Kingdom: A comparative examination of data from two surveys. Drug and Alcohol Dependence. 2020; 213:108080.

32. Straw S, Baig MW, Gillott R, Wu J, Witte KK, O'Regan DJ, et al. Long-term outcomes are poor in intravenous drug users following infective endocarditis, even after surgery. Clinical infectious diseases: an official publication of the Infectious Diseases Society of America. 2019: ciz869.

33. Summers PJ, Hellman JL, MacLean MR, Rees VW, Wilkes MS. Negative experiences of pain and withdrawal create barriers to abscess care for people who inject heroin. A mixed methods analysis. Drug and alcohol dependence. 2018; 190:200-8.

34. Monteiro J, Phillips KT, Herman DS, Stewart C, Keosaian J, Anderson BJ, et al. Self-treatment of skin infections by people who inject drugs. Drug and alcohol dependence. 2019; 206:107695-.

35. Serota DP, Bartholomew TS, Tookes HE. Evaluating differences in opioid and stimulant useassociated infectious disease hospitalizations in Florida, 2016-2017. Clinical infectious diseases: an official publication of the Infectious Diseases Society of America. 2020.

36. Asgeirsson $\mathrm{H}$, Thalme A, Weiland O. Low mortality but increasing incidence of Staphylococcus aureus endocarditis in people who inject drugs: Experience from a Swedish referral hospital. Medicine (Baltimore). 2016;95(49): e5617-e.

\section{Table}


Table 1

Baseline characteristics of the overall cohort $(\mathrm{N}=237)$

\begin{tabular}{|ll|}
\hline Characteristics & $\mathrm{n}(\%)$ or mean (SD) \\
\hline Age, years & $41.8(7.8)$ \\
\hline Sex, male & $186(78.5 \%)$ \\
\hline Spanish nationality & $137(57.8 \%)$ \\
\hline Mental disorder & $27(11.4 \%)$ \\
\hline Dose of methadone (milligrams) & $40(25)$ \\
\hline Time of drug consumption, years & $20(10)$ \\
\hline HIV status, positive & $76(32.3 \%)$ \\
\hline HCV antibodies, positive & $201(85.5 \%)$ \\
\hline Abbreviations: standard deviation (SD); hepatitis C virus (HCV); human immunodeficiency virus (HIV) \\
9No data was available for HCV RNA at the moment of initiation of the study. \\
\hline
\end{tabular}

\section{Figures}




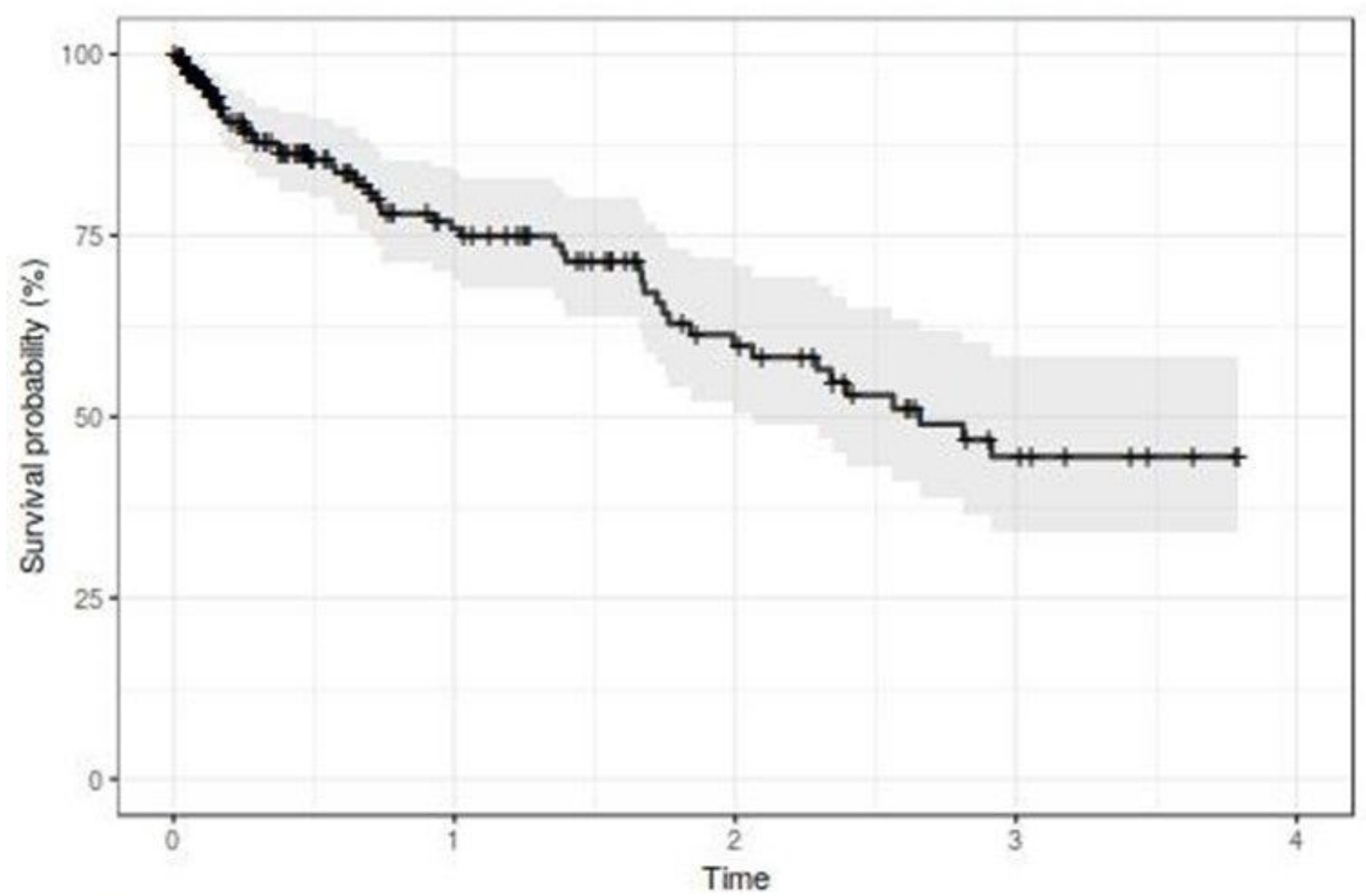

\section{Number at risk}

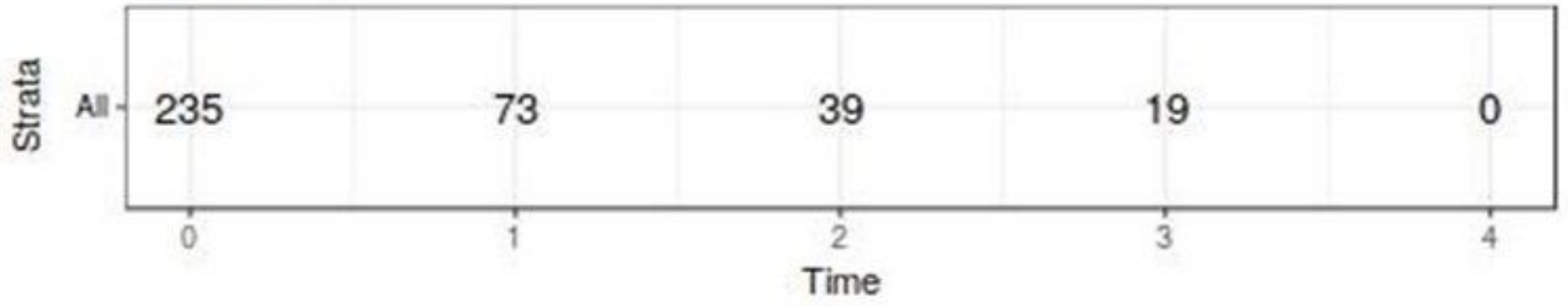

Figure 1

Kaplan-Meier curve estimating survival (free of any severe injection-related infections) of all patients in the cohort. 

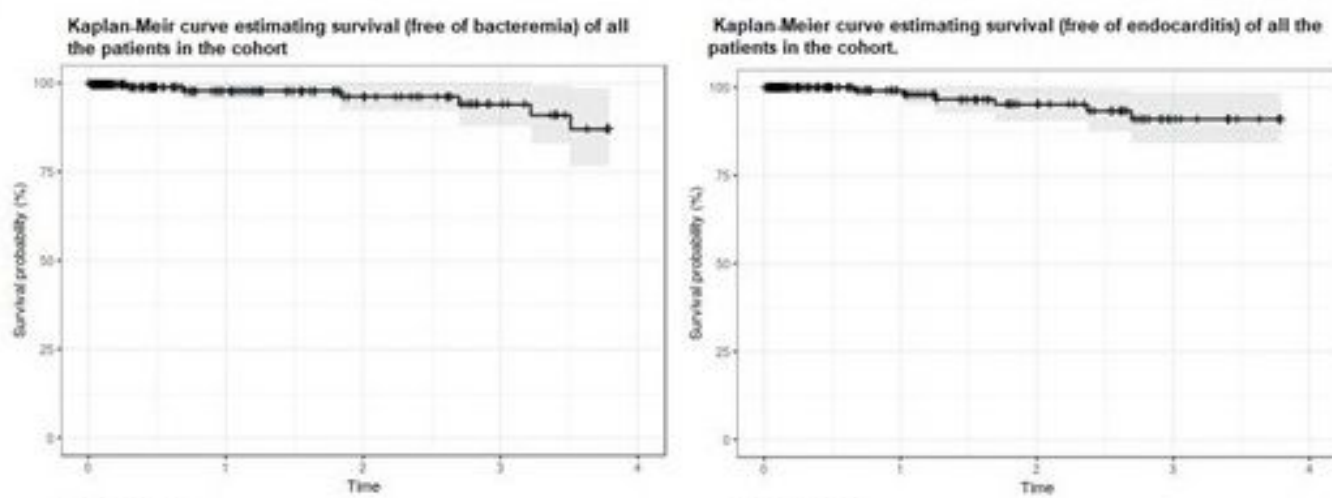
patients in the cohort.
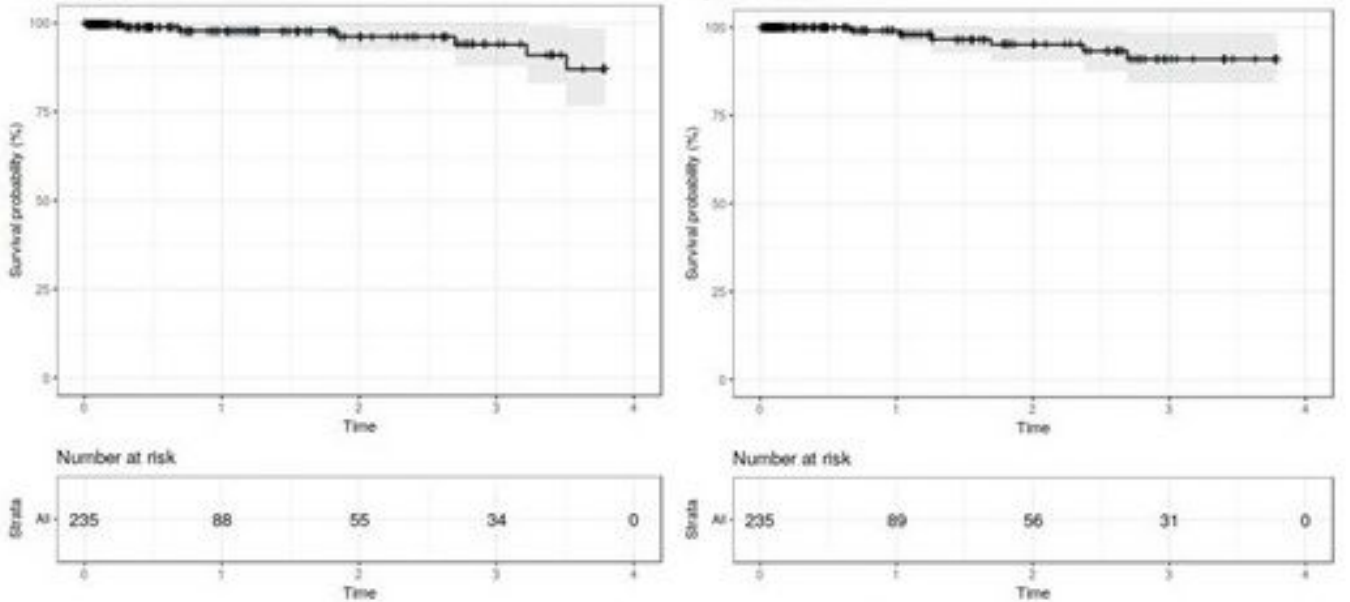

Number at nisk

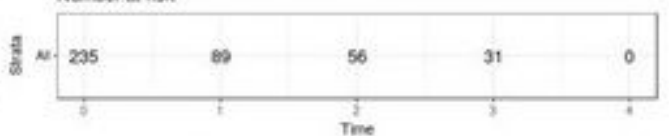

Kaplan Meier-curve estimating survival (tree of bactetemia with infection of noe-cardiac location) of all the patients in the cohort.

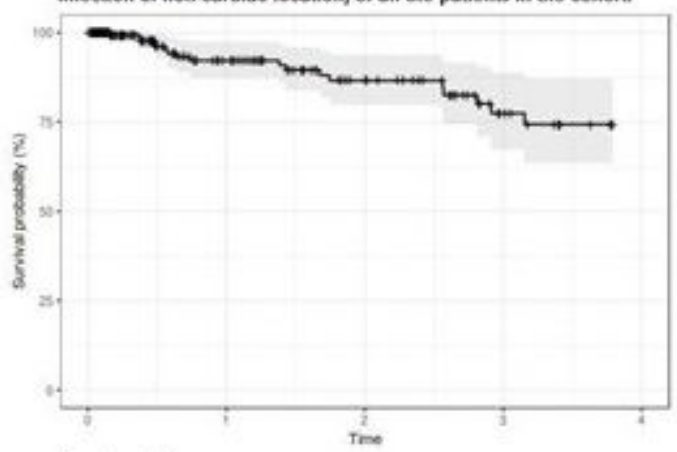

Kaplan-Meler Curve estimating survival ffree of skin and soft tissue Kaplan-Meler Curve estimating survival (fre
infections) of all the patients in the cohoth

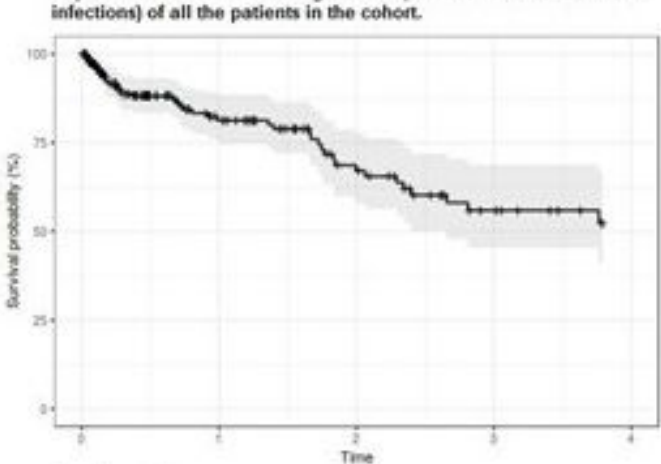

Number at tsok
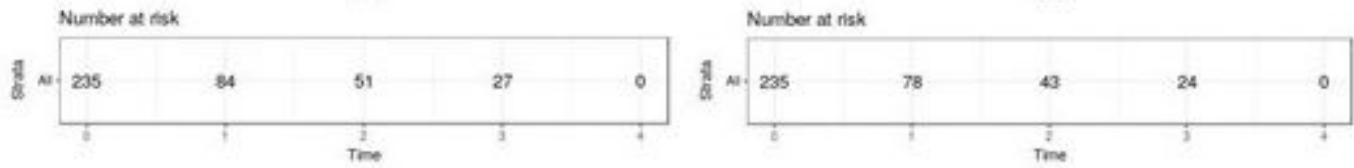

Figure 2

Kaplan-Meier curves stratified by type of severe injection-related infection of all patients in the cohort. 